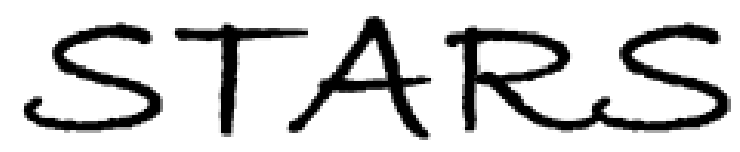

University of Central Florida

STARS

Faculty Bibliography 2000s

Faculty Bibliography

$1-1-2005$

\title{
Extraordinarily high-contrast and wide-view liquid-crystal displays
}

Qi Hong

University of Central Florida

Thomas X. Wu

University of Central Florida

Xinyu Zhu

University of Central Florida

Ruibo Lu

University of Central Florida

Shin-Tson Wu

University of Central Florida

Find similar works at: https://stars.library.ucf.edu/facultybib2000

University of Central Florida Libraries http://library.ucf.edu

This Article is brought to you for free and open access by the Faculty Bibliography at STARS. It has been accepted for inclusion in Faculty Bibliography 2000 s by an authorized administrator of STARS. For more information, please contactSTARS@ucf.edu.

\section{Recommended Citation}

Hong, Qi; Wu, Thomas X.; Zhu, Xinyu; Lu, Ruibo; and Wu, Shin-Tson, "Extraordinarily high-contrast and wide-view liquid-crystal displays" (2005). Faculty Bibliography 2000s. 5275.

https://stars.library.ucf.edu/facultybib2000/5275

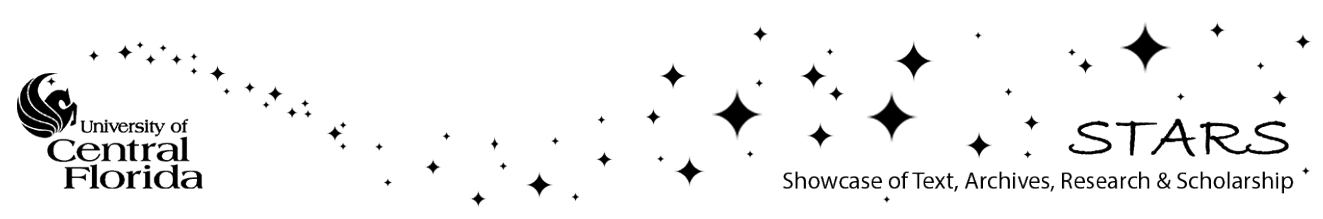




\title{
Extraordinarily high-contrast and wide-view liquid-crystal displays
}

\author{
Qi Hong, Thomas X. Wu, Xinyu Zhu, Ruibo Lu, and Shin-Tson Wua) \\ College of Optics and Photonics, University of Central Florida, Orlando, Florida 32816
}

(Received 18 October 2004; accepted 2 February 2005; published online 15 March 2005)

\begin{abstract}
A computer simulation model based on oblique-angle Jones matrix and Poincaré sphere is developed for optimizing the design of film-compensated multidomain vertical-alignment liquid crystal display (VA-LCD). According to this design, a contrast ratio higher than 10 000:1 is predicted over the entire $\pm 85^{\circ}$ viewing cone for the four-domain VA-LCD. Potential application for liquid-crystal display television is emphasized. (C) 2005 American Institute of Physics.
\end{abstract} [DOI: 10.1063/1.1887815]

High-contrast ratio and wide-viewing angle are critical requirements for liquid-crystal (LC) televisions. Presently, the view angle of a liquid-crystal display (LCD) is defined at isocontrast ratio greater than 10:1. A low contrast ratio implies a poor color rendering. Vertical alignment (VA) LCD exhibits an excellent contrast ratio at the normal viewing direction, weak color dispersion, and fast response time, ${ }^{1-4}$ however, its dark state light leakage at oblique angles is relatively large. Several analyses indicate that the dark state light leakage is determined by the polarization state of the outgoing beam before reaching the analyzer. ${ }^{5-10}$ To reduce dark state light leakage, different LC operation modes and compensation films have been proposed. For examples, the inplane-switching and optically compensated bend mode could exhibit a 300: 1 contrast ratio over the $\pm 80^{\circ}$ viewing cone. $^{5-9}$ However, for VA mode, the reported $\sim 100: 1$ isocontrast ratio is limited to the $\pm 50^{\circ}$ viewing cone. ${ }^{10}$ This is insufficient for TV applications. There is an urgent need to extend the high-contrast ratio to a wider-viewing cone.

In this letter, we optimize the design for a four-domain VA-LCD which shows an extraordinarily high-contrast ratio over the entire $\pm 85^{\circ}$ viewing cone. We begin with analyzing the polarization states inside the VA-LCD, and then optimizing the compensation films using the oblique-angle Jones matrix, and minimizing the dark state light leakage. Finally, we are able to obtain a VA-LCD with isocontrast ratio higher than 10000 : 1 over the $\pm 85^{\circ}$ viewing cone.

Figure 1 depicts the device configuration of a fourdomain VA-LCD with A-plate and C-plate compensation films. The absorption axes of polarizer and analyzer are in $0^{\circ}$ and $90^{\circ}$, respectively. Two A-plate films with equal thicknesses are laminated on the inner side of the crossed polarizers with their slow axes perpendicular to the absorption axes of the corresponding polarizers. Two equal thickness C-plate films are inserted between A-plate films and glass substrates. In the bright state, four domains are formed at $45^{\circ}, 135^{\circ}, 225^{\circ}$, and $315^{\circ}$. We use the finite difference method to simulate the bright state LC director distributions. ${ }^{11,12}$ The entire LCD is treated as multilayer device with each layer approximated by uniaxial anisotropic media. ${ }^{13}$ Assuming that the reflections between internal layers are negligible, the transmitted wave after the $m$ th layer is related to the incident wave as ${ }^{13}$

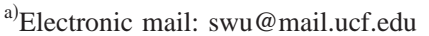

$$
\left[\begin{array}{c}
E_{\|} \\
E_{\perp}
\end{array}\right]_{m}=J_{m} \cdot J_{m-1} \cdots J_{2} \cdot J_{1} \cdot J_{\mathrm{ent}} \cdot\left[\begin{array}{c}
E_{\|} \\
E_{\perp}
\end{array}\right]_{\text {in }},
$$

where $J_{m}$ is the Jones matrix of the $m$ th layer and $J_{\text {ent }}$ is the correction matrix considering reflections on the air-polarizer interface. Approximating the propagating light inside the LCD by the plane wave, at the viewing angle $\theta$ and azimuthal angle of incident plane $\phi, J_{m}$ is obtained as ${ }^{14}$

$$
\begin{aligned}
J_{m}= & R(\psi) \cdot\left[\begin{array}{cc}
e^{-j(2 \pi / \lambda)\left(d / \cos \theta_{m}\right) n_{e}^{\prime}} & 0 \\
0 & e^{-j(2 \pi / \lambda)\left(d / \cos \theta_{m}\right) n_{o}^{\prime}}
\end{array}\right] \\
& \cdot R(-\psi), \\
R(\psi) & =\left[\begin{array}{cc}
\cos \Psi & -\sin \Psi \\
\sin \Psi & \cos \Psi
\end{array}\right],
\end{aligned}
$$

where $\lambda$ is the wavelength, $d$ is the thickness of the $m$ th layer, $\theta_{m}$ is the angle of light inside the $m$ th layer, and $n_{e}^{\prime}$ and $n_{o}^{\prime}$ are the refractive indices of the $m$ th layer media on the wave plane. ${ }^{1,13}$ As shown in Fig. 2, $\overline{O^{\prime} L}$ denotes the projection of the optical axis of the $m$ th layer $(\overline{O L})$ on the wave plane and $\Psi$ is the angle between $E_{\|}$and $\overline{O^{\prime} L}$, which is found to be

$$
\begin{aligned}
\Psi= & \operatorname{sign}\left(\sin \theta_{n e}-\frac{\cos \theta_{n e} \cos \left(\phi-\phi_{n e}\right)}{\tan \theta_{m}}\right) \\
& \times\left[\arcsin \left(\frac{\cos \theta_{n e} \sin \left(\phi-\phi_{n e}\right)}{\sin \Theta}\right)\right],
\end{aligned}
$$

where $\operatorname{sign}()$ is the sign function to distinguish angles

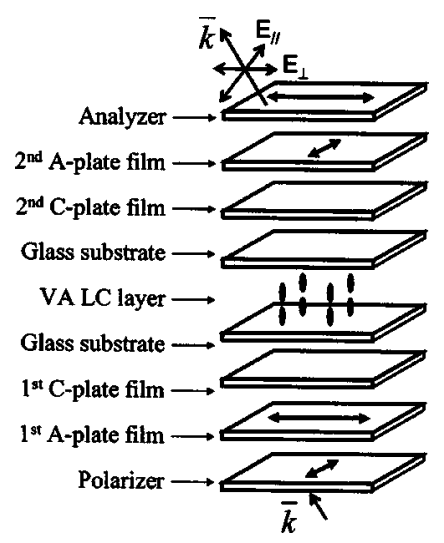

FIG. 1. Structure of VA-LCD for optimized design. The slow axis of each A-plate film is perpendicular to the absorption axis of the adjacent polarizer. 


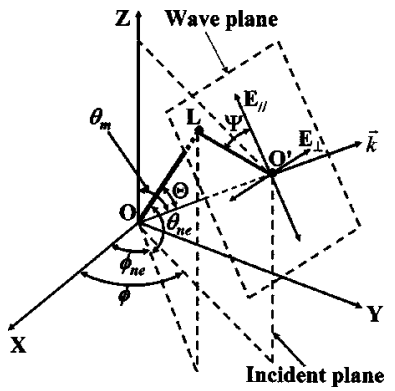

FIG. 2. The principal optical axis of the $m$ th layer $(\overline{O L})$ and its projection on the wave plane $\left(\overline{O^{\prime} L}\right)$.

greater than $90^{\circ}$, and $\theta_{n e}$ and $\phi_{n e}$ are the tilt and twist angles of $\overline{O L}$, respectively. In Fig. $2, \Theta$ is the angle between $\overline{O L}$ and wave vector $(\bar{k})$, which can be obtained from the dot product of $\overline{O L}$ and $\bar{k}$.

The state of polarization can be represented by Stokes parameters and plotted on Poincaré sphere, as shown in Fig. 3, after $E_{\|}$and $E_{\perp}$ are solved. ${ }^{14}$ Coordinates of Poincaré sphere are standard Stokes parameters $S_{1}, S_{2}$, and $S_{3}$. Due to the symmetry of VA-LCD in the dark state, we only investigate the states of polarization when $0^{\circ} \leqslant \phi \leqslant 90^{\circ}$. Results are applicable to $90^{\circ} \leqslant \phi \leqslant 360^{\circ}$. With the known $E_{\|}$and $E_{\perp}$, the bright and dark state transmittance can be obtained. ${ }^{13}$ Contrast ratio is defined as the ratio of bright state transmittance over dark state light leakage.

In Fig. 3, A denotes the state of polarization absorbed by the analyzer, $\mathbf{B}$ denotes the state of polarization in front of the analyzer, D denotes the state of polarization emerging from the VA LC layer, $\mathbf{G}$ denotes the state of polarization emerging behind the first A-plate film, and $\mathbf{P}$ denotes the state of polarization passing through the polarizer.

To analyze the effects of viewing angle on the states of polarization inside VA-LCD, we first obtain the Jones matrix of VA LC layer from Eq. (2) as

$$
\begin{aligned}
J= & e^{-j(\pi / \lambda)\left(d / \cos \theta_{\mathrm{LC}}\right)\left(n_{e}^{\prime}+n_{o}^{\prime}\right)} \\
& \times\left[\begin{array}{cc}
e^{-j(\pi / \lambda)\left(d / \cos \theta_{\mathrm{LC}}\right)\left(n_{e}^{\prime}-n_{o}^{\prime}\right)} & 0 \\
0 & e^{j(\pi / \lambda)\left(d / \cos \theta_{\mathrm{LC}}\right)\left(n_{e}^{\prime}-n_{o}^{\prime}\right)}
\end{array}\right] .
\end{aligned}
$$

As Eq. (4) shows, there is no coupling between $E_{\|}$and $E_{\perp}$ so that $S_{1}$ is not changed when light passes through the LC layer. However, the phase of $E_{\perp}$ leads the phase of $E_{\|}$for a positive LC $\left(n_{e}>n_{o}\right)$ and the difference increases with viewing angle $\theta$. Therefore, at oblique viewing angle, $S_{3}$ of $\mathbf{D}$ is

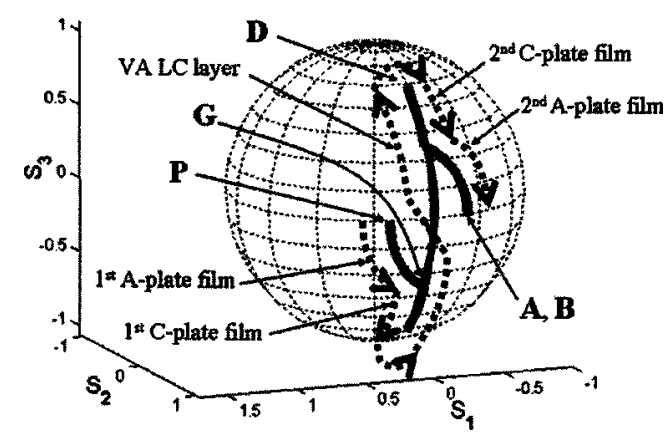

FIG. 3. States of polarization inside VA-LCD with optimal compensation films at $\theta=70^{\circ}, \phi=45^{\circ}$, and $\lambda=550 \mathrm{~nm} . \mathbf{P}, \mathbf{G}, \mathbf{D}, \mathbf{B}$, and $\mathbf{A}$ denote the state of polarization passing through polarizer, emerging from the first A-plate film, emerging from the VA LC layer, in front of the analyzer, and absorbed by the analyzer, respectively.

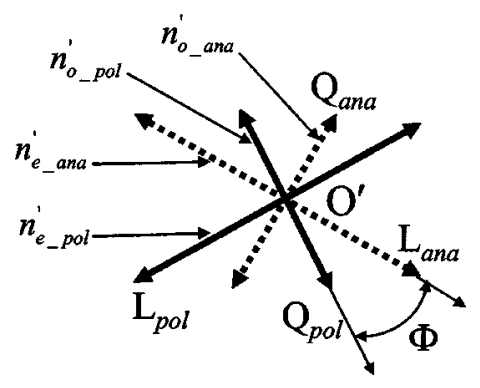

FIG. 4. Angle between the maximum transmission direction of the polarizer $\left({\overline{O^{\prime}}}_{\mathrm{pol}}\right)$ and the maximum absorption direction of the analyzer $\left(\bar{O}^{\prime} L_{\mathrm{ana}}\right)$. $\bar{O}^{\prime} Q_{\mathrm{pol}}$ is perpendicular to the maximum absorption direction of the polarizer $\left({\overline{O^{\prime} L}}_{\mathrm{pol}}\right)$.

greater than zero and increases with viewing angle for a linearly polarized input light. If there is no anisotropic media between the LC layer and analyzer, B equals D. Next, we model linear polarizer as lossy uniaxial anisotropic media. As Fig. 4 shows, on the wave plane, the maximum absorption direction of analyzer is along $\bar{O}^{\prime} L_{\text {ana }}$ and the maximum transmission direction of polarizer is along ${\overline{O^{\prime}}}_{\mathrm{pol}}$. Therefore, the difference between $S_{1}$ of $\mathbf{P}$ and $S_{1}$ of $\mathbf{A}$ depends on the angle between $\bar{O}^{\prime} Q_{\text {pol }}$ and $\bar{O}^{\prime} L_{\text {ana }}$, which is related to viewing angle $\theta$ and azimuthal angle $\phi$ as

$$
\begin{aligned}
\Phi= & \arctan \left(\frac{\cos \phi}{\sin \phi \cos \theta_{\mathrm{pol}}}\right)+\arctan \left(\frac{\sin \phi}{\cos \phi \cos \theta_{\mathrm{pol}}}\right) \\
& -90^{\circ} .
\end{aligned}
$$

Taking the derivative of $\Phi$ with respect to $\phi$ reveals that $\Phi$ reaches maximum at $\phi=45^{\circ}$. Next, taking the derivative of $\Phi$ with respect to $\theta_{\mathrm{pol}}$ at $\phi=45^{\circ}$ shows that $\Phi$ increases with viewing angle $\theta$. Therefore, the maximum of the difference between $S_{1}$ of $\mathbf{A}$ and $S_{1}$ of $\mathbf{P}$ occurs at maximal viewing angle when $\phi=45^{\circ}$. For a conventional VA-LCD, $S_{1}$ of $\mathbf{P}$ is not changed before the light reaches analyzer. Therefore, the $S_{1}$ of $\mathbf{B}$ equals the $S_{1}$ of $\mathbf{P}$.

For a conventional VA-LCD, the difference between $\mathbf{B}$ and $\mathbf{A}$ increases with viewing angle. If $\mathbf{B}$ and $\mathbf{A}$ are equal at a large oblique viewing angle when $\phi=45^{\circ}$, then the dark state light leakage would be greatly reduced at other viewing angles as well. Due to the symmetry of the device configuration shown in Fig. 1, the $S_{1}$ of $\mathbf{G}$ should satisfy the following condition

$$
S_{1 \_G}=\left(S_{1 \_P}+S_{1 \_A}\right) / 2 .
$$

Figure 3 illustrates the above relationship.

To design an A-plate film, we first find $E_{\|_{-} G}$ and $E_{\perp_{-} G}$ (after the first A-plate film) in terms of the A-plate film thickness $\left(d_{\text {A-plate }}\right)$ using Eq. $(1)$, provided that the polarizer thickness, refractive index, and the A-plate refractive index are known. Next, after $S_{1}$ of $\mathbf{A}$ and $S_{1}$ of $\mathbf{P}$ are solved, Eq. (6) can be expressed as

$$
S_{1 \_G}=\frac{\left(\left|E_{\|_{-} G}\right|^{2}-\left|E_{\perp_{-} G}\right|^{2}\right)}{\left(\left|E_{\|_{-} G}\right|^{2}+\left|E_{\perp_{-} G}\right|^{2}\right)}=\frac{\left(S_{1 \_}+S_{1 \_A}\right)}{2} .
$$

Simplification of Eq. (7) results in

$$
H_{1} \cdot \cos \left(K_{1} \cdot d_{\text {A-plate }}\right)-L_{1}=\left(S_{1 \_P}+S_{1_{-} A}\right) / 2,
$$

where constants $H_{1}, K_{1}$, and $L_{1}$ depend on the polarizer thickness and the refractive indices of the polarizer and the 


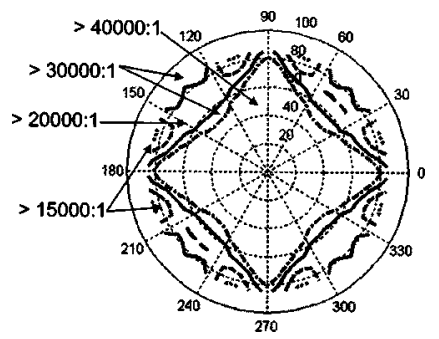

FIG. 5. Isocontrast ratio plot of the four-domain VA LCD with optimal compensation films optimized at $\theta=70^{\circ}$ and $\phi=45^{\circ}$.

A-plate film. Finally, from Eq. (8) we obtain $d_{\text {A-plate }}$ in the form of

$$
d_{\mathrm{A}-\text { plate }}=\frac{1}{K_{1}} \cdot \arccos \left(\frac{\left(S_{1 \_P}+S_{1-A}\right) / 2+L_{1}}{H_{1}}\right) .
$$

To design the C-plate film, we first note that for the optimum design, B satisfies conditions $S_{1_{-} B}=S_{1_{-} A}$ and $S_{3_{-} B}$ $=S_{3_{-} A}$. Similarly, we can find $E_{\|_{-B} B}$ and $E_{\perp_{-} B}$ (after the second A-plate film) in terms of the C-plate thickness $\left(d_{\mathrm{C} \text {-plate }}\right)$. Next, applying $S_{1 \_B}=S_{1 \_A}$ yields

$$
\frac{\left(\left|E_{\| \_B}\right|^{2}-\left|E_{\perp_{-} B}\right|^{2}\right)}{\left(\left|E_{\|_{-B} B}\right|^{2}+\left|E_{\perp_{-} B}\right|^{2}\right)}=S_{1_{-} A} \text {. }
$$

After simplifying Eq. (10), we derive the following expression

$$
H_{2} \cdot \cos \left(K_{2} \cdot d_{\text {C-plate }}\right)+L_{2} \cdot \sin \left(K_{2} \cdot d_{\text {C-plate }}\right)=S_{1 \_A},
$$

where constants $\mathrm{H}_{2}, \mathrm{~K}_{2}$, and $L_{2}$ depend on the thickness of polarizer, A-plate film, LC cell gap, and the refractive indices of polarizer, A-plate film, C-plate film, and LC material. Finally, from Eq. (11), we can find the thickness of each C-plate film $d_{\text {C-plate }}$.

Now, we apply the above methodology to design a VALCD shown in Fig. 1. The employed refractive indices of the polarizers, LC, A-plate, and C-plate are as follows: $n_{e_{-} \text {pol }}$ $=1.5+i \times 3.251 \times 10^{-3}$ and $n_{o-\mathrm{pol}}=1.5+i \times 2.86 \times 10^{-5}, n_{e_{-} \mathrm{LC}}$ $=1.5514$ and $n_{o_{-} \mathrm{LC}}=1.4737$ at $\lambda=550 \mathrm{~nm}, n_{e_{-} \mathrm{A}-\text { plate }}=1.5124$ and $n_{o_{-} \mathrm{A} \text {-plate }}=1.5089$, and $n_{e_{-} \mathrm{C} \text {-plate }}=1.5089$ and $n_{o_{-} \mathrm{C} \text {-plate }}$ $=1.5124$. The thickness of the polarizer is $150 \mu \mathrm{m}$ and LC cell gap is $4 \mu \mathrm{m}$.

We designed the compensation films at $\theta=70^{\circ}, \phi=45^{\circ}$, and $\lambda=550 \mathrm{~nm}$. From Eq. (9), we find the A-plate thickness $d_{\mathrm{A} \text {-plate }}=26.62 \mu \mathrm{m}$ and the $d \Delta n$ of each A-plate film is $93.17 \mathrm{~nm}$. Using Eq. (11), we find the thickness of each C-plate film $d_{\text {C-plate }}=21.54 \mu \mathrm{m}$. Therefore, the $d \Delta n$ of each C-plate film is $-75.39 \mathrm{~nm}$. With this design, in the dark state, the polarization state in front of the analyzer equals the polarization state absorbed by the analyzer at $\theta=70^{\circ}$ and $\phi$ $=45^{\circ}$. Therefore, a contrast ratio higher than $10000: 1$ over $\pm 85^{\circ}$ viewing cone is achieved, as shown in Fig. 5. The above ideal simulation results are obtained using the $4 \times 4$ matrix method. ${ }^{15}$ In a real display panel, the actual contrast ratio could be lowered because the above mentioned ideal parameters may not be precisely controlled. Moreover, the compensation film thickness variation and nonuniformity, LC alignment distortion near spacer balls, stress birefringence from films and substrates, and interface reflections between layers could also reduce the contrast ratio.

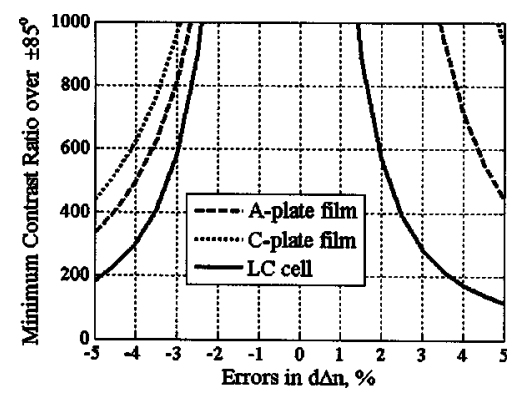

FIG. 6. Tolerance in the errors of an $d \Delta n$ of an A-plate film, C-plate film, and LC cell when the compensation films are optimized at $\theta=70^{\circ}$ and $\phi$ $=45^{\circ}$. The optimal $d \Delta n$ value of each A-plate film is $93.17 \mathrm{~nm},-75.39 \mathrm{~nm}$ for each C-plate film, and $310.8 \mathrm{~nm}$ for the LC cell.

Design tolerance is an important concern for display manufacturing. Figure 6 plots the minimum contrast ratio over the entire $\pm 85^{\circ}$ viewing cone if the $d \Delta n$ of A-plate film, C-plate film, and LC cell varies by $\pm 5 \%$ assuming that the compensation films are optimized at $\theta=70^{\circ}$ and $\phi=45^{\circ}$. From Fig. 6, the proposed VA-LCD is less sensitive to the $d \Delta n$ variation of the C-plate but more sensitive to the $d \Delta n$ variation of the LC cell. In the least favorable case (i.e., the LC $d \Delta n$ is $5 \%$ higher than the optimal value), the minimum contrast ratio is still higher than 100:1.

In conclusion, we demonstrate a wide-view VA LCD with a superb contrast ratio. We use the Poincaré sphere method to obtain the optimal compensation film parameters and then use $4 \times 4$ matrix method to calculate and plot the isocontrast contours. In the proposed design, a contrast ratio higher than 10000 : 1 is obtained over the entire $\pm 85^{\circ}$ viewing cone for the film-compensated four-domain VA LCD. The tolerance of the design is also investigated. Within $\pm 5 \%$ manufacturing margin, the contrast ratio maintains higher than 100:1.

The authors are indebted to the financial support from Toppoly Optoelectronics Corporation.

${ }^{1}$ S. T. Wu and D. K. Yang, Reflective Liquid Crystal Displays (Wiley, New York, 2001).

${ }^{2}$ S. H. Hong, Y. H. Jeong, H. Y. Kim, H. M. Cho, W. G. Lee, and S. H. Lee, J. Appl. Phys. 87, 8259 (2000).

${ }^{3}$ S. Kataoka, A. Takeda, H. Tsuda, Y. Koike, H. Inoue, T. Fujikawa, T. Sasabayashi, and K. Okamoto, Soc. Inf. Display Tech. Digest 37, 1066 (2001).

${ }^{4}$ K. Ohmuro, S. Kataoka, T. Sasaki, and Y. Koike, Soc. Inf. Display Tech. Digest 33, 845 (1997).

${ }^{5}$ Y. Saitoh, S. Kimura, K. Kusafuka, and H. Shimizu, Jpn. J. Appl. Phys., Part 1 37, 4822 (1998).

${ }^{6}$ T. Ishinabe, T. Miyashiita, and T. Uchida, Soc. Inf. Display Tech. Digest 36, 1094 (2000)

${ }^{7}$ H. Mori, Y. Itoh, Y. Nishiura, T. Nakamura, and Y. Shinagawa, Jpn. J. Appl. Phys., Part 1 36, 143 (1997).

${ }^{8}$ T. Miyashita, C. L. Kuo, M. Suzuki, and T. Uchida, Soc. Inf. Display Tech. Digest 31, 797 (1995).

${ }^{9}$ H. Mori, Y. Itoh, Y. Nishiura, T. Nakamura, and Y. Shinagawa, Soc. Inf. Display Tech. Digest 33, 941 (1997).

${ }^{10}$ J. Chen, K. H. Kim, J. J. Jyu, J. H. Souk, J. R. Kelly, and P. J. Bos, Soc.Inf. Display Tech. Digest 34, 315 (1998).

${ }^{11}$ J. E. Anderson, C. Titus, P. Watson, and P. J. Bos, Soc. Inf. Display Tech. Digest 38, 906 (2002).

${ }^{12}$ M. V. K. Chari and S. J. Salon, Numerical Methods in Electromagnetism (Academic, San Diego, 2000).

${ }^{13}$ A. Lien, Appl. Phys. Lett. 57, 2767 (1990).

${ }^{14}$ S. Huard, Polarization of Light (Wiley, New York, 1997).

${ }^{15}$ D. W. Berreman, J. Opt. Soc. Am. 62, 502 (1972). 